PROCEEDINGS OF THE

AMERICAN MATHEMATICAL SOCIETY

Volume 137, Number 11, November 2009, Pages 3593-3600

S 0002-9939(09)10014-X

Article electronically published on June 18, 2009

\title{
ON SINGULARITIES OF PRIMITIVE COHOMOLOGY CLASSES
}

\author{
MARK ANDREA A. DE CATALDO AND LUCA MIGLIORINI
}

(Communicated by Ted Chinburg)

\begin{abstract}
Green and Griffiths have introduced several notions of singularities associated with normal functions, especially in connection with middledimensional primitive Hodge classes. In this paper, by using the more elementary aspects of the Decomposition Theorem, we define global and local singularities associated with primitive middle-dimensional cohomology classes, and by using the Relative Hard Lefschetz Theorem, we show that these singularities detect the global and local triviality of the primitive class.
\end{abstract}

\section{INTRODUCTION}

Let $X^{2 n} \subseteq \mathbb{P}^{d}$ be a $2 n$-dimensional projective manifold embedded in projective space. The study of the hyperplane sections of $X$ has proved to be very fruitful classically, as well as in present times. In the context of this paper, the evendimensional case is the critical case.

Following Poincaré and Lefschetz, Griffiths has introduced ([7]) normal functions, i.e. certain holomorphic cross sections of the bundle of intermediate Jacobians of the hyperplane sections of $X$ over the complement of the dual variety $\check{X}$ inside the dual space $\mathbb{P}^{\vee}$. The original hope, modeled on Lefschetz's proof of the $(1,1)$-Theorem, had been to start with a primitive, i.e. killed by cupping with a hyperplane class, integral $(n, n)$-class in middle cohomology, extract a highly transcendental normal function, and then build an algebraic cycle out of it, thus establishing the truth of the Hodge Conjecture ( $\mathrm{HC})$.

As is well-known, this approach meets a very serious difficulty due to the wellknown failure of Jacobi inversion in dimension higher than two.

In [8], Thomas has shown that the Hodge Conjecture (HC) is equivalent to the existence of special hyperplane sections; see Remarks 3.7 and 3.9.

In [6], Green and Griffiths have introduced several notions of singularities of normal functions, especially in connection with middle-dimensional primitive Hodge classes, and have tied these notions to the HC. The notions are an attempt at codifying the behavior of normal functions near the dual variety of singular hyperplane sections. Roughly speaking, the $\mathrm{HC}$ is equivalent to the nonvanishing of these singularities at some point of $\check{X}$. As is well-known, by virtue of standard inductive arguments, the middle-dimensional case is the critical one.

The various notions of singularities in 6] reflect the subtle geometries that one needs to explore to further this point of view.

Received by the editors June 11, 2008, and, in revised form, February 12, 2009

2000 Mathematics Subject Classification. Primary 14D06, 14D07.

(C)2009 American Mathematical Society 
In this paper, we concentrate on what is perhaps the simplest of these notions and propose an essentially elementary definition (cf. Definition 3.3) of what we call the Green-Griffiths singularity associated with a primitive middle-dimensional class on a projective manifold. There are a local (i.e. having to do with a single hyperplane section) and a global version (i.e. having to do with all hyperplane sections).

Our definition is possible in view of the most basic properties of the perverse filtration that can be read off directly from the Decomposition Theorem of Beilinson, Bernstein, Deligne and Gabber 1 .

The universal hyperplane family $\pi: \mathfrak{X} \rightarrow \mathbb{P}^{\vee}$ is a proper map with equidimensional fibers which is smooth away from the dual variety $\check{X} \subseteq \mathbb{P}^{\vee}$. The cohomology $H(\mathfrak{X}, \mathbb{Q})$ finds itself filtered by the perverse filtration, and this filtration is splittable by virtue of the Decomposition Theorem. This remains true replacing $\mathbb{P}^{\vee}$ with any open set.

We prove, using the Relative Hard Lefschetz Theorem [1] (a kind of symmetry present on $H(\mathfrak{X}, \mathbb{Q})$, which is the relative analogue of the classical Hard Lefschetz Theorem), Propositions 3.6 and 3.8 which establish that the local/global triviality of the primitive class is detected precisely by the Green-Griffiths invariants.

Section 2 is for preliminaries on the Decomposition Theorem. Section 3 contains the results of this paper mentioned above.

By using M. Saito's theories of mixed Hodge modules and admissible normal functions, the preprint 2] introduces, among other things, a notion of singularity that essentially coincides with ours. We hope that the two different points of view can both be useful for further geometric investigations.

We have reached the conclusions contained in $\$ 3$ in the Spring of 2007 during our visit at I.A.S., Princeton. We thank Phil Griffiths for inspiring conversations. We thank the referee for correcting some inaccuracies.

\section{Decomposition theorem FormulÆ}

In this section we collect the facts we need from the Decomposition Theorem in [1.

2.1. Setup. We work with rational cohomology. Let:

- $X^{2 n} \subseteq \mathbb{P}^{d}$ be a smooth, irreducible and projective manifold of dimension $2 n$;

- $\mathcal{L}$ be the hyperplane bundle;

$-\zeta \in H^{2 n}(X)$ be a cohomology class;

- there be the universal hyperplane family (for simplicity, we write $\mathbb{P}$ also for $\mathbb{P}^{\vee}$ )

$$
X \stackrel{q}{\longleftarrow} \mathfrak{X} \stackrel{\pi}{\longrightarrow} \mathbb{P}^{d}, \quad \operatorname{dim} \mathfrak{X}=2 n-1+d,
$$

and we have the hyperplane sections as fibers

$$
\mathfrak{X}_{p}:=\pi^{-1}(p), \quad \operatorname{dim} \mathfrak{X}_{p}=2 n-1
$$

The main objects of investigation here are the classes

$$
q^{*} \zeta \in H^{2 n}(\mathfrak{X}), \quad\left(q^{*} \zeta\right)_{\mid \mathfrak{X}_{p}}=\zeta_{\mid \mathfrak{X}_{p}} \in H^{2 n}\left(\mathfrak{X}_{p}\right) .
$$

Let $Z$ be a variety, and let $L$ be a local system on a dense open subset $U \subseteq Z_{\text {reg }}$. The intersection cohomology complex $I C_{Z}(L)$ is a complex of sheaves on $Z$. Its cohomology sheaves satisfy

$$
\mathcal{H}^{l}\left(I C_{Z}(L)\right)=0, \quad l \notin[-\operatorname{dim} Z,-1] .
$$


We have the intersection cohomology groups

$$
I H^{k}(Z, L):=\mathbb{H}^{k-\operatorname{dim} Z}\left(Z, I C_{Z}(L)\right)
$$

Clearly,

$$
I H^{k}(Z, L)=0, \quad k \notin[0,2 \operatorname{dim} Z] .
$$

2.2. The decomposition theorem for $\pi$. The Decomposition Theorem for $\pi$ : $\mathfrak{X} \rightarrow \mathbb{P}^{d}$ gives a noncanonical decomposition

$$
\phi: \bigoplus_{i \in \mathbb{Z}} \bigoplus_{j \in \mathbb{N}} I C\left(L_{i j}\right)[-i-(2 n-1+d)] \simeq R \pi_{*} \mathbb{Q},
$$

where $L_{i j}$ is a local system on the codimension $j$ stratum $S_{d-j} \subseteq \mathbb{P}^{d}$. Strata are not connected, so that by $I C$ here we mean the direct sum of the $I C$ 's on the connected components of the same dimension.

The $L_{i 0}$ are the local systems on $S_{d} \subseteq \mathbb{P}^{d} \backslash X^{\vee}$ :

$$
L_{i 0}=R^{2 n-1+i}:=\left(R^{2 n-1+i} \pi_{*} \mathbb{Q}\right)_{\mid S_{d}} .
$$

In what follows, the pedantic notation is to make the formulæ ready to use later. We have a noncanonical decomposition for the cohomology groups

$$
H^{l}(\mathfrak{X})=\phi\left(\bigoplus_{i \in \mathbb{Z}} \bigoplus_{j \in \mathbb{N}} I H^{(l-2 n)-j-(i-1)}\left(\overline{S_{d-j}}, L_{i j}\right)\right),
$$

one for cohomology sheaves

$$
R^{l} \pi_{*} \mathbb{Q}=\phi\left(\bigoplus_{i \in \mathbb{Z}} \bigoplus_{j \in \mathbb{N}} \mathcal{H}^{(l-2 n)-(d-j)-j-(i-1)}\left(I C\left(L_{i j}\right)\right)\right)
$$

and one for the cohomology groups

$$
\left(R^{l} \pi_{*} \mathbb{Q}\right)_{p}=H^{l}\left(\mathfrak{X}_{p}\right)=\phi\left(\bigoplus_{i \in \mathbb{Z}} \bigoplus_{j \in \mathbb{N}} \mathcal{H}^{(l-2 n)-(d-j)-j-(i-1)}\left(I C\left(L_{i j}\right)\right)_{p}\right) .
$$

2.3. Definition of the filtrations on $H(\mathfrak{X})$ and $H\left(\mathfrak{X}_{p}\right)$. These filtrations are discussed and used in our paper [3. The theory of perverse sheaves filters the groups $H(\mathfrak{X})$. The Decomposition Theorem makes this more visible.

Remark 2.1 (Reminder on the perverse filtration). The perverse filtration on $H(\mathfrak{X})$ is by Hodge substructures, and it coincides, up to some shifts, with the monodromy filtration associated with the nilpotent cup-product action of $q^{*} L$ on $H(\mathfrak{X})[3$. A splitting $\phi_{\mathcal{L}}$ in the category of Hodge structures exists, as shown in [4], but we do not need it here (see Remark 3.5). Over the regular part of $\pi$, the perverse filtration coincides, up to renumbering, with the filtration coming from the Leray spectral sequence.

The perverse filtration on $H^{l}(\mathfrak{X})$ is the increasing filtration indexed by $i \in \mathbb{Z}$ :

$$
H_{\leq i}^{l}(\mathfrak{X}):=\phi\left(\bigoplus_{i^{\prime} \leq i} \bigoplus_{j \in \mathbb{N}} I H^{(l-2 n)-j-\left(i^{\prime}-1\right)}\left(\overline{S_{d-j}}, L_{i^{\prime} j}\right)\right) \subseteq H^{l}(\mathfrak{X}) .
$$

The perverse filtration is independent of the splitting $\phi$. The graded pieces $H_{i}^{l}(\mathfrak{X})$ are canonically isomorphic to

$$
H_{i}^{l}(\mathfrak{X})=\bigoplus_{j \in \mathbb{N}} I H^{(l-2 n)-j-(i-1)}\left(\overline{S_{d-j}}, L_{i j}\right) .
$$


The decomposition $\phi$ induces, in the same way, an increasing filtration on the stalks which we call the induced filtration (also independent of $\phi$ ),

$$
H_{\leq i}^{l}\left(\mathfrak{X}_{p}\right)=\phi\left(\bigoplus_{i^{\prime} \leq i} \bigoplus_{j \in \mathbb{N}} \mathcal{H}^{(l-2 n)-(d-j)-j-\left(i^{\prime}-1\right)}\left(I C\left(L_{i^{\prime} j}\right)\right)_{p} \subseteq H^{l}\left(\mathfrak{X}_{p}\right),\right.
$$

with graded pieces canonically isomorphic to

$$
H_{i}^{l}\left(\mathfrak{X}_{p}\right)=\bigoplus_{j \in \mathbb{N}} \mathcal{H}^{(l-2 n)-(d-j)-j-(i-1)}\left(I C\left(L_{i j}\right)\right)_{p} .
$$

The restriction map $r: H(\mathfrak{X}) \rightarrow H\left(\mathfrak{X}_{p}\right)$ is filtered and strict (obvious since the map is a direct sum map) with respect to the perverse and induced filtrations:

$$
r: H_{\leq i}(\mathfrak{X}) \rightarrow H_{\leq i}\left(\mathfrak{X}_{p}\right) .
$$

If we fix a small neighborhood $U \subseteq \mathbb{P}^{d}$ of $p$ and set $\mathfrak{X}_{U}:=\pi^{-1}(U)$, then we have the filtered isomorphisms

$$
r: H_{\leq i}\left(\mathfrak{X}_{U}\right) \simeq H_{\leq i}\left(\mathfrak{X}_{p}\right)
$$

\section{The Green-Griffiths Singularity}

3.1. Bound on the filtrations on $H(\mathfrak{X})$ and $H\left(\mathfrak{X}_{p}\right)$. In order to define the Green-Griffiths singularity we need (ii) below.

Lemma 3.1. (i)

$$
H_{\leq 1}^{2 n}(\mathfrak{X})=H^{2 n}(\mathfrak{X}), \quad H_{\leq 1}^{2 n}\left(\mathfrak{X}_{p}\right)=H^{2 n}\left(\mathfrak{X}_{p}\right) .
$$

(ii) If $\zeta \in H^{2 n}(X)$ is primitive, then

$$
q^{*} \zeta \in H_{\leq 0}^{2 n}(\mathfrak{X}), \quad \zeta_{\mid \mathfrak{X}_{p}} \in H_{\leq 0}^{2 n}\left(\mathfrak{X}_{p}\right) .
$$

Proof. By virtue of the obvious vanishing (2.3) in negative degrees, the graded piece (2.10) becomes

$$
H_{i}^{2 n}(\mathfrak{X})=\bigoplus_{j \in \mathbb{N}} I H^{-j-(i-1)}\left(\overline{S_{d-j}}, L_{i j}\right)=0, \quad \forall i \geq 2 .
$$

This holds also for $\mathfrak{X}_{U}$. In view of (2.13), this proves (i).

The graded piece

$$
H_{1}^{2 n}(\mathfrak{X})=\bigoplus_{j \in \mathbb{N}} I H^{-j}\left(\overline{S_{d-j}}, I C\left(L_{1 j}\right)\right)=I H^{0}\left(\mathbb{P}^{d}, L_{10}\right) .
$$

By (2.5), $L_{10}=R^{2 n}$ is the local system on the dense stratum of $\mathbb{P}^{d}$ corresponding to the variation of $H^{2 n}\left(\mathfrak{X}_{\eta}\right)$, where $\mathfrak{X}_{\eta}$ is a smooth hyperplane section. The group in question is just the space of global invariants. Since we are assuming that $\zeta$ is primitive, $\zeta_{\mid \mathfrak{x}_{\eta}}=0$ defines the zero section in this group and (ii) follows. 
3.2. Definition of the Green-Griffiths invariant. We have the decompositions: (2.6) for $H(\mathfrak{X})$ (noncanonical), (2.10) for the graded $H_{i}(\mathfrak{X})$ (canonical), (2.8) for $H\left(\mathfrak{X}_{p}\right)$ (noncanonical) and $(2.12)$ for the graded $H_{i}\left(\mathfrak{X}_{p}\right)$ (canonical).

Let $\zeta \in H^{2 n}(X)$. There is the noncanonical decomposition associated with $\phi$ :

$$
q^{*} \zeta=\phi\left(\sum_{i j}\left[q^{*} \zeta\right]_{i j}\right), \quad \zeta \mid \mathfrak{X}_{p}=\phi\left(\sum_{i j}\left[\zeta_{\mid \mathfrak{x}_{p}}\right]_{i j}\right),
$$

where the terms $[-]_{i j}$ depend on $\phi$.

Let $\zeta \in H^{2 n}(X)$ be primitive. Then, by Lemma 3.1 the terms $[-]_{0}$ and $[-]_{0 j}$ are well-defined, independently of $\phi$ :

$$
\begin{gathered}
{\left[q^{*} \zeta\right]_{0}=\sum_{j \in \mathbb{N}}\left[q^{*} \zeta\right]_{0 j} \in H_{0}^{2 n}(\mathfrak{X})=\bigoplus_{j \in \mathbb{N}} I H^{-j+1}\left(\overline{S_{d-j}}, I C\left(L_{0 j}\right)\right),} \\
{\left[\zeta{\mid \mathfrak{X}_{p}}_{p}\right]_{0}=\sum_{j \in \mathbb{N}}\left[\zeta{\mid \mathfrak{X}_{p}}_{0 j}\right]_{0 j} \in H_{0}^{2 n}\left(\mathfrak{X}_{p}\right)=\bigoplus_{j \in \mathbb{N}} \mathcal{H}^{-d+1}\left(\operatorname{IC}\left(L_{0 j}\right)\right)_{p} .}
\end{gathered}
$$

As in the proof of Lemma 3.1 complemented by the support conditions (2.1) and (2.3), the terms with $j \geq 2$ are zero:

$$
\begin{aligned}
{\left[q^{*} \zeta\right]_{0} } & =\left[q^{*} \zeta\right]_{00}+\left[q^{*} \zeta\right]_{01}, \\
{\left[\zeta \mid \mathfrak{X}_{p}\right]_{0} } & =\left[\zeta_{\mid \mathfrak{X}_{p}}\right]_{00}+\left[\zeta \mid \mathfrak{X}_{p}\right]_{01},
\end{aligned}
$$

where, remembering that our notation calls for $I C_{Z}(L)$ to have cohomology sheaves in the interval $[-\operatorname{dim} Z,-1]$ we write explicitly:

$$
\left[\zeta_{\mid \mathfrak{x}_{p}}\right]_{00} \in \mathcal{H}^{-d+1}\left(I C\left(R^{2 n-1}\right)\right)_{p}, \quad\left[\zeta_{\mid \mathfrak{x}_{p}}\right]_{01} \in \mathcal{H}^{-d+1}\left(I C\left(L_{01}\right)\right)_{p} .
$$

Remark 3.2. The local system $L_{01}$ is usually defined on the regular part of the dual variety $X^{\vee} \subseteq \mathbb{P}^{d}$. If we take the embedding associated with $m \mathcal{L}, m \gg 0$, then the local system $L_{01}=0$. This follows from [SGA 7.2, XVIII. 5.3.5 ("Condition A") and 6.4 ("Condition A" is verified for $m \gg 0$ )]. In fact, the stalk of $L_{01}$ at a general point of the dual hypersurface measures the failure of the adjunction map 5.3.2 (loc. cit.) to be an isomorphism (it is surjective for Lefschetz pencils). This can also be seen by using the Clemens-Schmid sequence.

Definition 3.3. Let $\zeta \in H^{2 n}(X)$ be a primitive class.

The global Green-Griffiths invariant $s(\zeta)$ is defined to be

$$
s(\zeta):=\left[q^{*} \zeta\right]_{00} \in I H^{1}\left(\mathbb{P}^{d}, I C\left(R^{2 n-1}\right)\right) .
$$

The local Green-Griffiths invariant $s(\zeta)_{p}$ is defined to be

$$
s(\zeta)_{p}:=\left[\zeta_{\mid \mathfrak{X}_{p}}\right]_{00} \in \mathcal{H}^{-d+1}\left(I C\left(R^{2 n-1}\right)\right)_{p} .
$$

Clearly, these invariants depend on the embedding.

Remark 3.4. From the conditions of support of $I C$, it follows that the locus

$$
\operatorname{Sing}(\zeta):=\left\{p \in \mathbb{P}^{d} \mid s(\zeta)_{p} \neq 0\right\}
$$

is of codimension at least two.

Remark 3.5. The Hodge-theoretic splitting $\phi_{\mathcal{L}}$ of 4 shows that $H_{0}^{2 n}(\mathfrak{X})$ is endowed with a natural pure Hodge structure so that if $\zeta$ is a Hodge class, then so are $\left[q^{*} \zeta\right]_{0}$ and $s(\zeta)$. Our paper [4 does not afford local results. However, using M. Saito's general theory of mixed Hodge modules, one can reach similar conclusions for $\left[\zeta_{\mid \mathfrak{x}_{p}}\right]_{0}$ and $s(\zeta)_{p}$. 
3.3. The classes $\left[q^{*} \zeta\right]_{0}$ and $\left[\zeta_{\mid x_{p}}\right]_{0}$ detect global/local triviality. Given a primitive class $\zeta \in H^{2 n}(X)$, the class $q^{*} \zeta \in H_{\leq 0}^{2 n}$ and it defines a canonical element $\left[q^{*} \zeta\right]_{0} \in H_{0}^{2 n}(\mathfrak{X})$. The same holds for $\zeta_{\mid \mathfrak{X}_{p}} \in H_{\leq 0}^{2 n}\left(\mathfrak{X}_{p}\right)$ and $\left[\zeta_{\mid \mathfrak{X}_{p}}\right]_{0} \in H_{0}^{2 n}\left(\mathfrak{X}_{p}\right)$.

The following proposition ensures that, given any embedding $|\mathcal{L}|$, the global/local triviality of primitive classes is detected by the global/local classes $[-]_{0}$.

This is a simple consequence of the Relative Hard Lefschetz Theorem [1. In particular, we only need that cupping with the relatively ample line bundle $q^{*} \mathcal{L}$ is injective on $H_{\leq-1}(\mathfrak{X})$ and on $H_{\leq-1}\left(\mathfrak{X}_{p}\right)$ (see [4]).

Proposition 3.6. Let $\zeta \in H^{2 n}(X)$ be primitive.

(i) The class $\zeta=0 \operatorname{IFF}\left[q^{*} \zeta\right]_{0}=0$.

(ii) The class $\zeta_{\mid \mathfrak{X}_{p}}=0 \operatorname{IFF}\left[\zeta_{\mathfrak{X}_{p}}\right]_{0}=0$.

Proof. We prove (i). The proof for $(i i)$ is analogous. One direction is trivial. Let $\zeta \neq 0$, so that $q^{*} \zeta \neq 0$. If $\left[q^{*} \zeta\right]_{0}=0$, then $q^{*} \zeta \in H_{\leq-1}^{2 n}(\mathfrak{X})$. By the Relative Hard Lefschetz Theorem, the cup product with $q^{*} \mathcal{L}$ is injective on $H_{\leq-1}^{2 n}(\mathfrak{X})$, contradicting $q^{*}(\mathcal{L} \cdot \zeta)=0$.

Remark 3.7 (Relation with the Hodge Conjecture). Thomas [8] has proved that the Hodge conjecture is equivalent, given an arbitrary middle-dimensional primitive Hodge class $\zeta$, to the existence of $m \gg 0$ such that there exists $p \in$ $|m \mathcal{L}|$ with $\mathfrak{X}_{p}$ nodal and $\zeta_{\mid \mathfrak{X}_{p}} \neq 0$. If we drop the nodal requirement, the resulting statement is of course still true. Because of primitivity, the hypersurface must be singular, and nodality is an interesting improvement. See also Remark 3.9.

3.4. The local Green-Griffiths invariants detects global/local triviality. The Green-Griffiths invariant captures the primitive class; i.e. we have the following, where by $m \gg 0$ we mean that we replace the embedding given by $|\mathcal{L}|$ with the one given by $|m \mathcal{L}|$, with $m \gg 0$ :

Proposition 3.8. Let $\zeta \in H^{2 n}(X)$ be primitive.

(i) The class $\zeta=0$ iff $s(\zeta)=0$.

(ii) Let $m \gg 0$. The class $\zeta_{\mid \mathfrak{X}_{p}}=0$ iff $s(\zeta)_{p}=0$.

Proof. By Remark 3.2 and by (3.3) and (3.4), we have that for $m \gg 0$ :

$$
s(\zeta)_{p}=\left[\zeta_{\mid \mathfrak{X}_{p}}\right]_{00}=\left[\zeta_{\mid \mathfrak{X}_{p}}\right]_{0},
$$

and we apply Proposition 3.6 to deduce (ii). The proof of (i) is identical (except for the fact that the result holds for every embedding).

Remark 3.9. Proposition 3.8 implies that one can state R. Thomas' result using $\left[\zeta \mid \mathfrak{x}_{p}\right]_{0}$, or $s(\zeta)_{p}$, instead of $\zeta_{\mid \mathfrak{x}_{p}}$. We do not need the Hodge-theoretic nature of $\phi_{\mathcal{L}}$ to prove the resulting statement. As is pointed out by B. Totaro, one uses $\zeta_{\mid x_{p}}$ and Deligne's mixed Hodge structures.

3.5. Further characterizations of vanishing of $s(\zeta)_{p}$. In this section we clarifiy the relation between $s(\zeta)_{p}$ and two other invariants associated with $\zeta$.

The natural map $\mathbb{Q} \mathfrak{X}_{p}\left[\operatorname{dim} \mathfrak{X}_{p}\right] \rightarrow I C_{\mathfrak{X}_{p}}$ induces a map $H^{2 n}\left(\mathfrak{X}_{p}\right) \rightarrow I H^{2 n}\left(\mathfrak{X}_{p}\right)$. By [4, Theorem. 3.2.1, the kernel of this map is precisely $W_{2 n-1} H^{2 n}\left(\mathfrak{X}_{p}\right)$. Since $\zeta_{\mid \mathfrak{X}_{p}}$ is of type $(n, n)$ for the mixed Hodge structure, it is not in the kernel, and we have the following (cf. 6], Theorem. 2.ii ): 
Corollary 3.10. Let $\zeta \in H_{\mathbb{Q}}^{n, n}(X)$ be a primitive Hodge class on $X$.

The class $\left[\zeta_{\mid \mathfrak{X}_{p}}\right]_{0}=0$ iff $\zeta_{\mid \mathfrak{X}_{p}}=0$ in $I^{2 n}\left(\mathfrak{X}_{p}\right)=I H_{2 n-2}\left(\mathfrak{X}_{p}\right)$.

Let $m \gg 0$. The Green-Griffiths invariant $s(\zeta)_{p}=0$ iff $\zeta_{\mid \mathfrak{X}_{p}}=0$ in $I H^{2 n}\left(\mathfrak{X}_{p}\right)=$ $I H_{2 n-2}\left(\mathfrak{X}_{p}\right)$.

It is possible to give a more precise characterization for the locus in which the Green-Griffiths invariant does not vanish, which appears as a natural generalization of the condition for the (non)extendibility of a normal function in [5]. We need the preliminary

Lemma 3.11. Let $U$ be a contractible neighborhood of $0 \in \mathbb{C}^{d}$, let $D \ni 0$ be a divisor, and let $L$ be a local system on $U \backslash D$. Then $\mathcal{H}^{1-d}(I C(L))_{0}$ injects naturally in $H^{1}(U \backslash D, L)$.

Proof. Let $U^{*}=U_{d} \subseteq U_{d-1} \subseteq \ldots \subseteq U_{0}=U$ be the ascending chain of open subsets $U_{l}=\coprod_{l^{\prime} \geq l} S_{l^{\prime}}$ associated with a stratification of $\left(\mathbb{C}^{d}, D\right)$, and denote by $j_{l}: U_{l+1} \rightarrow U_{l}$ the corresponding imbeddings. We have the well-known formula

$$
I C_{U}(L):=\tau_{\leq-1} R j_{0 *}\left(\ldots\left(\tau_{\leq-d+1} R j_{d-2 *}\left(\tau_{\leq-d} R j_{d-1 *} L[d]\right)\right) \ldots\right) .
$$

Since the truncations relative to $j_{i}$ for $i \leq d-2$ are in degree greater than or equal to $1-d$, and $\tau_{\leq-d} R j_{d-1_{*}} L[d]=\left(j_{d-1_{*}} L\right)[d]$, setting $J: U_{d-1} \rightarrow U$ we have

$$
\left.\mathcal{H}^{1-d}(I C(L))_{0}=\mathbb{H}^{1-d}\left(U, R J_{*} j_{d-1_{*}} L\right)[d]\right)=H^{1}\left(U_{d-1}, j_{d-1_{*}} L\right) .
$$

The latter cohomology group is the term $E_{2}^{10}$ in the Grothendieck spectral sequence for $\mathbb{H}^{1}\left(U_{d-1}, R j_{d-1_{*}} L\right)=H^{1}\left(U^{*}, L\right)$. The statement follows from the edge sequence.

Recall our notation $\mathfrak{X}_{U}=\pi^{-1}(U)$.

Corollary 3.12. Let $U$ be a contractible neighborhood of $p$ and $U^{*}=U \backslash U \cap X^{\vee}$. Let $m \gg 0$. Then $s(\zeta)_{p}=0$ iff $q^{*} \zeta_{\mid \mathfrak{X}_{U^{*}}}=0$ in $H^{2 n}\left(\mathfrak{X}_{U^{*}}\right)$.

Proof. Let $u: U^{*} \rightarrow U$ be the open imbedding. The map $\pi: \pi^{-1}\left(U^{*}\right) \rightarrow U^{*}$ is smooth so that we have, by Deligne's theorem, the splitting on the right-hand side below:

$$
R \pi_{*} \mathbb{Q}_{\mathfrak{x}_{U}} \longrightarrow R u_{*} u^{*} R \pi_{*} \mathbb{Q}_{\mathfrak{X}_{U}} \simeq \bigoplus R u_{*} R^{l} \pi_{*} \mathbb{Q}_{\mathfrak{x}_{U^{*}}}[-l] .
$$

The statement follows from 3.8 (ii) and 3.11 applied to $L=R^{2 n-1}=R^{2 n-1} \pi_{*} \mathbb{Q}_{\mathfrak{X}_{U^{*}}}$.

\section{REFERENCES}

[1] A.A. Beilinson, J.N. Bernstein, P. Deligne, "Faisceaux pervers", Astérisque 100, Soc. Math. France, Paris, 1982. MR751966 (86g:32015)

[2] P. Brosnan, H. Fang, Z.O. Nie, G. Pearlstein, "Singularities of admissible normal functions," preprint, arXiv:0711.0964, 2007.

[3] M. de Cataldo, L. Migliorini, "The Hodge Theory of Algebraic maps," Ann. Sci. École Norm. Sup. (4) 38 (2005) no. 5, 693-750. MR2195257(2007a:14016)

[4] M.A. de Cataldo, L. Migliorini, "Hodge-theoretic aspects of the decomposition theorem," Algebraic Geometry - Seattle 2005, Proc. Sympos. Pure Math., vol. 80, Part 2, Amer. Math. Soc., Providence, RI, 2009, 489-504.

[5] F. El Zein, S. Zucker, "Extendability of normal functions associated to algebraic cycles," Chapter XV of Topics in transcendental algebraic geometry, Ann. of Math. Studies, vol. 106, Princeton University Press, 1984. MR756857 
[6] M. Green, P. Griffiths, "Algebraic cycles and singularities of normal functions," Algebraic Cycles and Motives (Grenoble, 2006), London Math. Soc. Lecture Notes Series, vol. 343, Cambridge University Press, 2007, 206-263. MR:2385303 (2009b:14013)

[7] P. Griffiths, "Periods of integrals on algebraic manifolds. III. Some global differentialgeometric properties of the period mapping," Inst. Hautes Études Sci. Publ. Math. No. 38, 1970, 125-180. MR0282990 (44:224)

[8] R.P. Thomas, "Nodes and the Hodge conjecture," J. Algebraic Geom. 14 (2005), no. 1, 177-185. MR2092131 (2005h:14024)

Department of Mathematics, SUny at Stony Brook, Stony Brook, New York 11794

Dipartimento di Matematica, Università di Bologna, 40126 Bologna, Italy 